\title{
April 2014 Pulmonary Case of the Month: DIP-What?
}

\author{
Lewis Wesselius MD \\ Department of Pulmonary Medicine \\ Mayo Clinic Arizona \\ Scottsdale, AZ
}

\section{History of Present IIIness}

A 53 year old woman from Indiana was seen who had a history of nonproductive cough for several years. She had a prior diagnosis of asthma but continued to have cough despite asthma treatment. She was also treated for gastroesophageal reflux and had a Nissen fundoplication. This resolved in some improvement in the cough. In May 2013 she noted increasing dyspnea on exertion.

An echocardiogram was performed which was notable for a $16 \%$ left ventricular ejection fraction. A thoracic CT demonstrated some nodules and a question was raised of sarcoidosis. She was admitted to a hospital in Indiana and had a biventricular pacemaker placed. Bronchoscopy with transbronchial biopsy was performed with no diagnostic findings. No granulomas were seen on the biopsy. Bronchoalveolar lavage showed a CD4/CD8 ration of 0.84 . Optic nerve swelling was noted at that time. Due to the cardiac, pulmonary, and optic nerve findings a clinical diagnosis of sarcoidosis with a dilated cardiomyopathy was made and she was treated with prednisone initially, then a combination of prednisone and methotrexate.

\section{$\mathrm{PMH}, \mathrm{FH}, \mathrm{SH}$}

Her past medical history was as above and family history was noncontributory. She does not smoke or drink.

\section{Medications}

- Methotrexate $15 \mathrm{mg}$ weekly

- Prednisone $5 \mathrm{mg}$ daily

- Furosemide $40 \mathrm{mg}$ daily

- Potassium chloride 20 meq daily

\section{Physical Examination}

Afebrile. SpO2 96\% on room air. The physical exam was unremarkable. 
Which of the following should be performed at this time?

1. Pulmonary function testing

2. Repeat echocardiogram

3. Repeat thoracic CT scan

4. 1 and 3

5. All of the above 


\section{Correct! \\ 5. All of the above}

This is not a course typical of sarcoidosis although sarcoidosis can have a myriad of presentations. The echocardiogram, thoracic CT scan and echocardiogram are all part of the routine work up for a heart transplant. A repeat of the echocardiogram confirmed that the left ventricular ejection was low at $23 \%$. Pulmonary function testing is below.

Table 1. Pulmonary function testing.

\begin{tabular}{|c|c|c|c|c|c|c|}
\hline PFT & FVC & FEV1 & FEV1/FVC & TLC & RV & DLco \\
\hline$\%$ predicted & $46 \%$ & $35 \%$ & 0.60 & $71 \%$ & $107 \%$ & $52 \%$ \\
\hline
\end{tabular}

Abbreviations:

- FEV1-forced expiratory volume in the first 1 second of expiration

- FVC-forced vital capacity

- FEV1/FVC-ratio of FEV1 to FVC

- TLC-total lung capacity

- RV-residual volume

- DLco-diffusing capacity for carbon monoxide

Which of the following is the best interpretation of the pulmonary function testing?

1. Mixed obstructive and restrictive lung disease with reduced DLco

2. Normal

3. Obstructive lung disease with reduced DLco

4. Restrictive lung disease with reduced DLco

5. Restrictive lung disease with normal DLco 


\section{Correct! \\ 1. Mixed obstructive and restrictive lung disease with moderately reduced DLco.}

Although there are several protocols for interpreting pulmonary function testing, most are similar and the American Thoracic Society/European Thoracic Society guidelines are perhaps the most commonly used (1). The types of ventilatory defects are summarized in table 2.

Table 2. Summary of types of ventilatory defects and their diagnoses (1).

\begin{tabular}{|l|l|}
\hline Abnormality & Diagnosis \\
\hline Obstruction & $\begin{array}{l}\text { Reduced FEV1/FVC, 5th percentile of predicted (usually } \\
<70 \%)\end{array}$ \\
\hline Restriction & TLC $<70 \%, 5$ th percentile of predicted (usually $<70 \%)$ \\
\hline Mixed defect & $\begin{array}{l}\text { FEV1/VC and TLC ,5th percentile of predicted (usually }<70 \% \\
\text { for both) }\end{array}$ \\
\hline
\end{tabular}

A thoracic CT was performed (Figure 1).
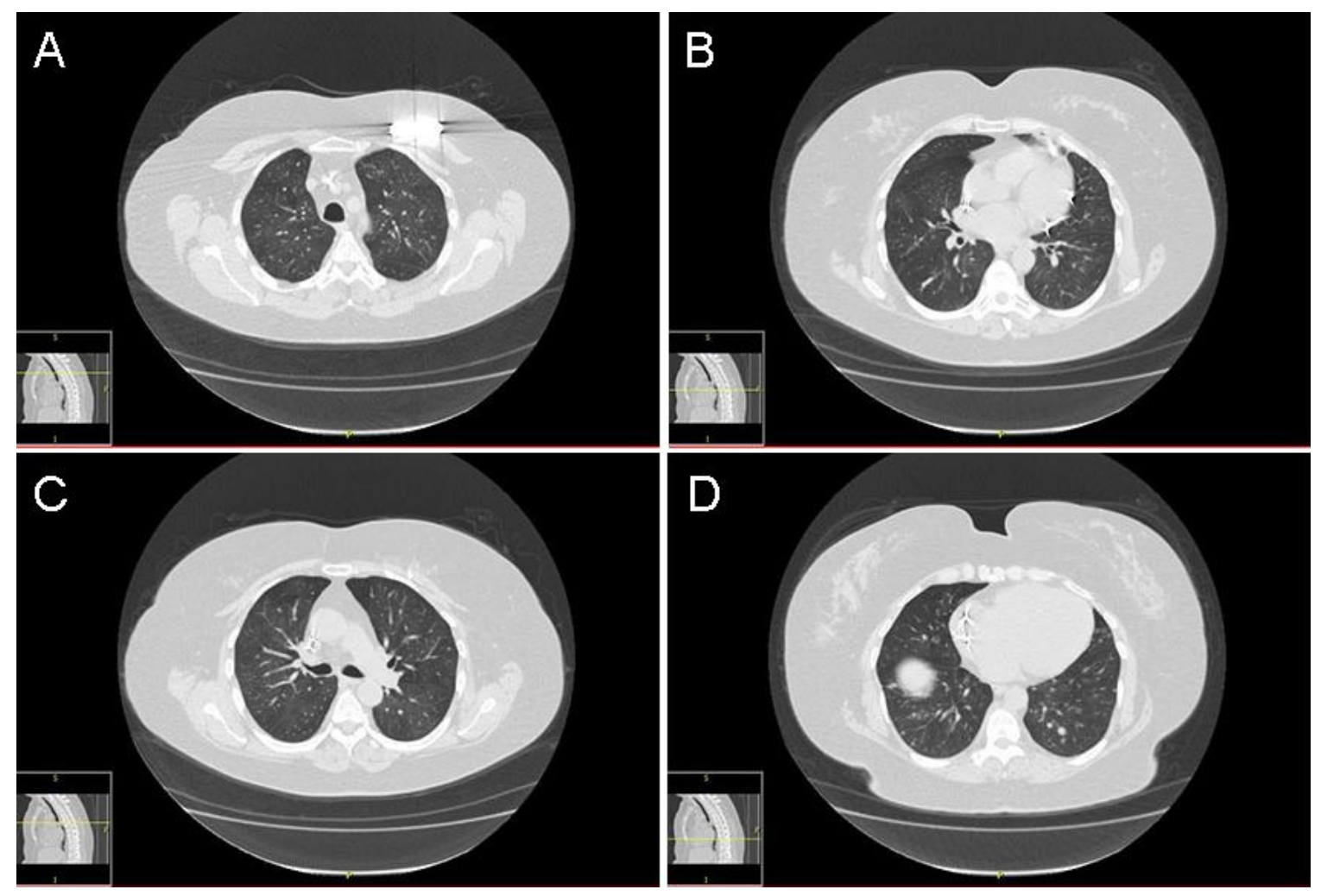

Figure 1. Representative lung windows from the thoracic CT scan. 
Which of the following is the best interpretation of the thoracic CT scan?

1. "Crazy paving"

2. Diffuse consolidation

3. Pulmonary edema

4. Scattered small lung nodules

5. Solitary nodule in the left lower lobe 


\section{Correct! \\ 4. Scattered small lung nodules}

There are numerous small lung nodules on the thoracic CT scan. "Crazy" paving is when the thoracic CT shows multifocal areas of ground-glass opacity (defined as areas of increased lung attenuation without obscuration of the underlying vessels and airway walls), associated with smooth interlobular septal thickening, with a sharp, non-anatomic demarcation between normal and abnormal lung (2).

What should be done next to diagnose her lung disease?

1. Needle biopsy of the lung

2. Repeat bronchoscopy with transbronchial biopsy

3. Serial thoracic CT scans

4. Thoracic CT angiography

5. Video-assisted thorascopic lung biopsy (VATS) 


\section{Correct! \\ 5. Video-assisted thorascopic lung biopsy (VATS)}

The transplant team was trying to make a decision whether to perform a heart transplant and so making a diagnosis was imperative. It would not be wrong to repeat the bronchoscopy with transbronchial biopsy but the rationale was that the previous biopsy was nondiagnostic and a repeat would probably also be nondiagnostic. Needle biopsy is usually for solitary pulmonary nodules and thoracic CT angiography is usually for abnormalities of the pulmonary blood vessels such as pulmonary embolism, AV malformation, etc.

The results of the histology from the VATS are shown in Figure 2.

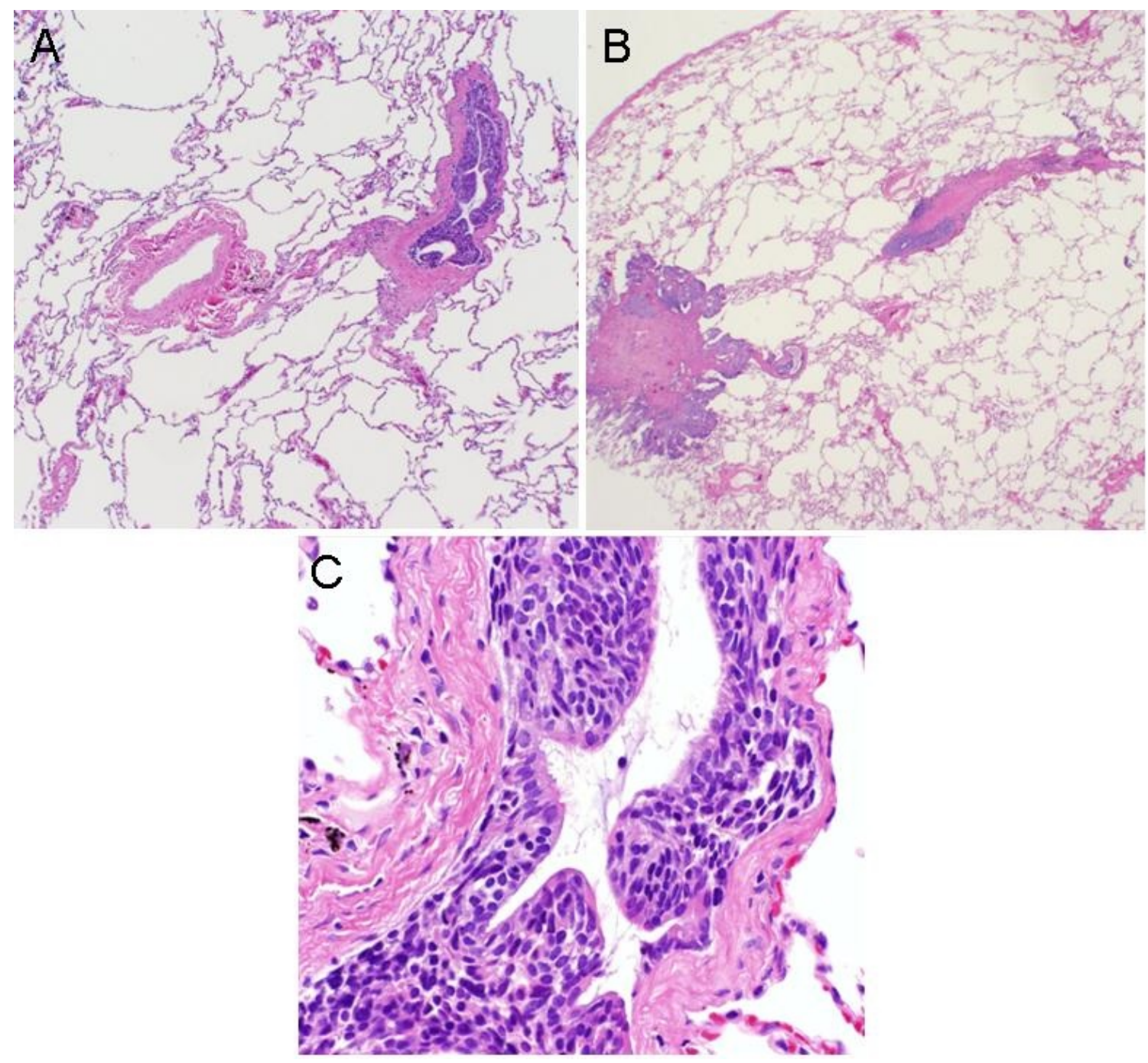

Figure 2. Panels $A$ and $B$ : Low power view of $H$ \& $E$ stained lung biopsy. Panel C: Higher power view of panel A. 
Which of the following describe the biopsy?

1. Areas of normal lung

2. Hyperplasia of some bronchioles with intraluminal narrowing

3. Small areas of cellular proliferation compatible with tumorlets

4. All of the above

5. None of the above 


\section{Correct! \\ 4. All of the above}

There are areas of normal lung interspersed with proliferation of cells both in small bronchi and bronchioles and outside the airway. For those outside the airway proliferating in small nodules the term tumorlet is appropriate. The proliferating cells stained with synaptophysin, also known as the major synaptic vesicle protein $\mathrm{p38}$, a marker for neuroendocrine cells. The histology is characteristic of diffuse idiopathic pulmonary neuroendocrine cell hyperplasia (DIPNECH).

DIPNECH was described by Sam Aguayo (3) now at the Phoenix VA while he was at the University of Colorado. Recently Nassar et al. (4) from the Mayo Clinic Arizona described 24 cases in the literature and added an additional case. The majority of patients presenting with DIPNECH are middle-aged females with symptoms of cough and dyspnea; obstructive abnormalities on pulmonary function testing; and radiographic imaging showing pulmonary nodules, groundglass attenuation, and bronchiectasis. In general, the clinical course remains stable; however, progression to respiratory failure does occur. Long-term followup studies and the best treatment remains unknown.

The present patient was declined for heart transplant and returned to Indiana on inhaled corticosteroids.

\section{References}

1. Pellegrino R, Viegi G, Brusasco V, et al. Interpretative strategies for lung function tests. Eur Respir J. 2005;26(5):948-68. [CrossRef] [PubMed]

2. Gotway MB. August 2012 imaging case of the month. Southwest J Pulm Crit Care 2012;5:74-81. Available at:

http://www.swjpcc.com/imaging/2012/8/5/august-2012-imaging-case-of-themonth.html

3. Aguayo SM, Miller YE, Waldron JA Jr., Bogin RM, Sunday ME, Staton GW Jr, Beam WR, King TE Jr. Brief report: idiopathic diffuse hyperplasia of pulmonary neuroendocrine cells and airways disease. N Engl J Med 1992;327:1285-8. [CrossRef] [PubMed]

4. Nassar AA, Jaroszewski DE, Helmers RA, Colby TV, Patel BM, Mookadam F. Diffuse idiopathic pulmonary neuroendocrine cell hyperplasia: a systematic overview. Am J Respir Crit Care Med. 2011;184(1):8-16. [CrossRef] [PubMed] 